\title{
Model-Driven Adaptation for Plastic User Interfaces
}

\author{
Jean-Sébastien Sottet ${ }^{1}$, Vincent Ganneau ${ }^{1,2}$, Gaëlle Calvary ${ }^{1}$, \\ Joëlle Coutaz ${ }^{1}$, Alexandre Demeure ${ }^{1}$, Jean-Marie Favre ${ }^{1}$, and Rachel Demumieux ${ }^{2}$ \\ ${ }^{1}$ Université Joseph Fourier, Laboratoire LIG, BP 53, 38041 Grenoble Cedex 9, France \\ \{Jean-Sebastien.Sottet, Vincent.Ganneau, Gaelle.Calvary, \\ Joelle.Coutaz, Alexandre.Demeure, Jean-Marie.Favre\} @imag.fr \\ ${ }^{2}$ France Télécom R\&D, 2 avenue Pierre Marzin, 22307 Lannion Cedex France \\ \{Vincent.Ganneau, Rachel.Demumieux\} @orange-ftgroup.com
}

\begin{abstract}
User Interface (UI) plasticity denotes UI adaptation to the context of use (user, platform, physical and social environments) while preserving usability. In this article, we focus on the use of Model-Driven Engineering and demonstrate how the intrinsic flexibility of this approach can be exploited by designers for UI prototyping as well as by end-users in real settings. For doing so, the models developed at design-time, which convey high-level design decisions, are still available at run-time. As a result, an interactive system is not limited to a set of linked pieces of code, but is a graph of models that evolves, expresses and maintains multiple perspectives on the system from top-level tasks to the final UI. A simplified version of a Home Heating Control System is used to illustrate our approach and technical implementation.
\end{abstract}

Keywords: User interface plasticity, user interface adaptation, context aware systems, Model-Driven Engineering.

\section{Introduction}

User Interface (UI) plasticity denotes the capacity for user interfaces to adapt to the context of use while preserving usability [34]. The context of use is a structured information space whose finality is to inform the adaptation process. It includes a model of the user who is intended to use (or is actually using) the system, the social and physical environments where the interaction is supposed to take place (or is actually taking place), and the platform to be used (or is being used). The latter covers the set of computing, sensing, communication, and interaction resources that bind together the physical environment with the digital world. Usability expresses the useworthiness of the system: the value that this system has in the real world [6].

From the software perspective, UI plasticity goes far beyond UI portability and UI translation. Software adaptation has been addressed using many approaches over the years, including Machine Learning [22], Model-Driven Engineering (MDE) [5, 11, 18, 20, 23, 25, 31, 32], and Component-oriented services [29]. Our approach to the problem of UI plasticity is based on the following observations. First, every paradigm 
has its own merits targeted at specific requirements. Thus, in an ever-changing world, one single approach is doomed to failure. Second, software tools and mechanisms tend to make a dichotomy between the development stage and the run-time phase making it difficult to articulate run-time adaptation based on semantically rich designtime descriptions.

Our approach to UI plasticity is to bring together Model-Driven Engineering (MDE) and Service Oriented Approach (SOA) within a unified framework that covers the development stage of interactive systems as well as the run-time phase. Our intention is to demonstrate how the intrinsic flexibility of this approach can be usefully exploited for UI prototyping by designers as well as by end-users (if appropriately encapsulated). In this article, we focus on the MDE aspects of our solution space. The principles are presented in Section 3, followed in Section 4 by the description of an early implementation, that combines MDE and SOA. To illustrate the discussion, we use a simplified version of a Home Heating Control System (HHCS). This running case study is depicted in Section 2.

\section{HHCS: A Simplified Home Heating Control System}

HHCS (Home Heating Control System) enables users to control the temperature of the home using different interaction resources. A typical task consists in checking and setting room temperature. As illustrated in Fig. 1, the graphical rendering of the user interface for this task depends on the screen size (as in 1-b and 1-d), on the set of screens that can be used simultaneously (e.g., 1-a versus 1-e), as well as on the set of usability properties that the designers have considered as central. Switching between these UIs is performed at run-time under human control (i.e. by the end-user in the real setting and/or the designer while prototyping the UI).

The five UIs of Fig. 1 are functionally equivalent: they support the same set of tasks (i.e. to access a set of rooms and, if needed, to set the room temperature between $15^{\circ} \mathrm{C}$ and $18^{\circ} \mathrm{C}$ ). Some UIs are centralized on a single screen but simultaneously mapped onto different displays whose size differs (Fig. 1 a-b-c-d). Conversely, in Fig. 1-e, the UI is distributed over two displays: the UI that corresponds to the task "Select room" is mapped onto the PDA, whereas the PC is used to set the temperature.

In addition, these UIs do not satisfy the same set of usability properties. In particular, prompting (cf. Bastien-Scapin's framework [3]), prevention against errors, and minimal actions, are not equally supported. In Fig. 1-a, the range of values of the room temperature is not observable. As a result, prompting is not fully supported. Prevention against errors is improved in Fig. 1-c by using a menu list while Fig. 1-b improves minimal actions by eliminating the articulatory task for selecting a room.

In the rest of the article, we present the underlying technology and its principles that enable end-users and/or designers to control the final UI (FUI). 


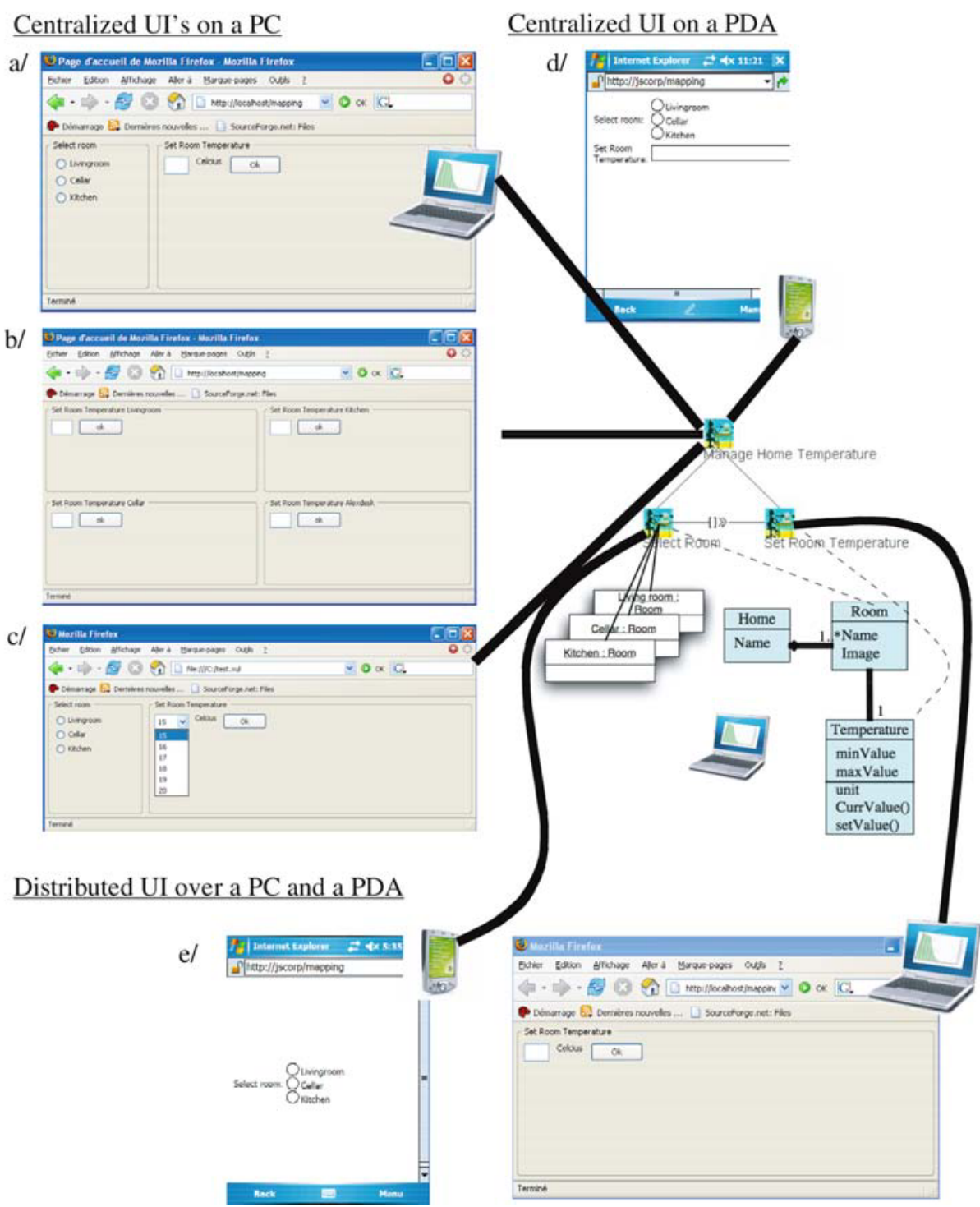

Fig. 1. Five final UIs for HHCS resulting from different mappings (depicted as black lines) between the platforms available (PC and PDA) and high-level models (e.g., task and concepts).

\section{Principles}

Early work in the automatic generation of UIs as well as more recent work in UI adaptation $[5,11,18,20,23,25,31,32]$ adheres only partially to the MDE principles. Our approach differs from previous work according to the following five principles. 


\subsection{Principle \#1: An Interactive System Is a Graph of Models}

An interactive system is a graph of models that are compliant to meta-models [32]. This graph, which expresses and maintains multiple perspectives on the system, is available at design-time as well as at run-time (see Fig. 2). Thus, an interactive system is not limited to executable code: it also includes models that convey high-level design decisions. As shown in Fig. 2, a UI may include a task model, a concept model, a workspace model (also called AUI for Abstract UI), and an interactor model (i.e., CUI for Concrete UI) linked by mappings ${ }^{l}$. In turn, the UI components are mapped onto items of the Functional Core of the interactive system, whereas the CUI elements (the interactors) are mapped onto the input and output (I/O) devices of the platform. Mapping between interactors and I/O devices supports the explicit expression of centralized versus distributed user interfaces.

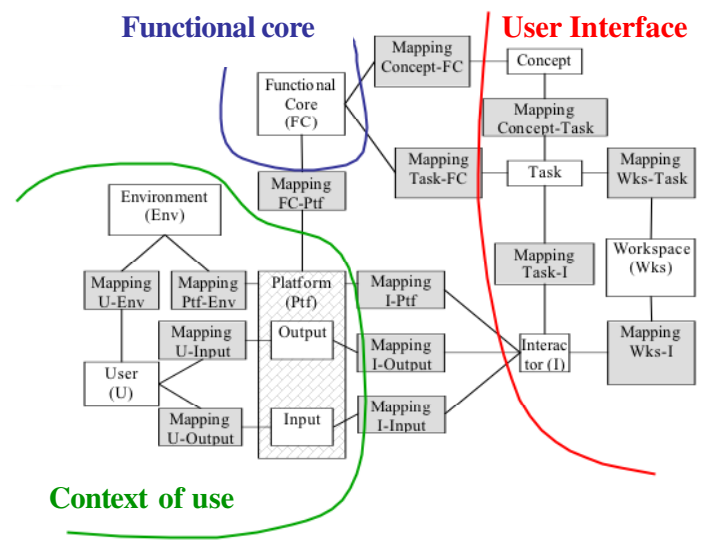

Fig. 2. An interactive system is a graph of models related by mappings. White boxes denote the models whereas lines and grey boxes denote mappings.

Traditional model-based approaches to UI generation focus on the final UI, targeted at specific technological spaces [13] (e.g., XUL, Swing, etc.). Forward engineering is applied, starting from the task and concept models, loosing the intermediate models as well as the transformations used in the generation process. Consequently, "the connection between specification and final result can be quite difficult to control and to understand" [19]. In our approach, transformations are key.

\subsection{Principle \#2: Transformations Are Models}

In the context of MDE, a transformation is the production of a set of target models from a set of source models, according to a transformation definition. In turn, a transformation definition is a set of transformation rules that together describe how source

\footnotetext{
${ }^{1}$ In mathematics, a mapping is "a rule of correspondence established between tow sets that associates each member of the first set with a single member of the second" [The American Heritage Dictionary of the English Language, 1970, p. 797].
} 
models are transformed into target models. In particular, our transformation rules make explicit the usability properties they satisfy (cf. Principle \#3).

By promoting transformations as models, transformations can also be transformed, and frequent transformations (such as transforming sub-tasks into pull-down menus) can serve as patterns in a library. As a result, we alleviate the development and maintenance cost (by the way of a library of re-usable transformations), we support rapid prototyping based on the comparative evaluation ${ }^{2}$ of UIs produced with different transformations, we improve run-time flexibility (since transformations, which are available at run-time, can be transformed). For example, the UIs of HHCS differ from the application of different transformations. Examples will be presented in more detail in Section 4.1.

\subsection{Principle \#3: The Choice of Usability Frameworks Is Left Opened}

A large number of usability frameworks have been proposed to better understand and measure the usability of interactive systems. These include: Shackel [28], Dix et al. [10], Nielsen [21], Preece [26], Schneiderman [30], Constantine and Lockwood [7], Van Welie et al. [35], as well as Seffah et al. [27] who propose QUIM, a unifying roadmap to reconcile existing frameworks. More specific frameworks are proposed for web engineering (Montero et al. [17]), or for specific domains (for instance, military applications). Closely related to UI plasticity, Lopez-Jacquero et al. propose a refinement of Bastien-Scapin's framework, as a usability guide for UI adaptation [15].

Because moving to an unfamiliar set of tools would impose a high threshold on HCI and software designers, we promote an open approach that consists in choosing the appropriate usability framework for eliciting the properties that must, should or may be satisfied by transformations [33]. For HHCS, we have used Bastien-Scapin's framework. The transformation of a usability framework into a digital model is performed according to Principle \#4.

\subsection{Principle \#4: Humans Are Kept in the Loop}

HCI design methods produce a large body of contemplative models (i.e. models that cannot be processed by a machine) such as storyboards, and mock-ups. These models are useful reference material during the design process. On the other hand, because they are contemplative, they can only be transformed manually into productive models (i.e. models that can be processed by a machine). Manual transformation supports creative inspiration, but is prone to wrong interpretation and to loss of key information. To address this problem, we accept to support a mix of automated, semiautomated, and manually performed transformations. Semi-automated and manual transformations may be performed by designers and/or end-users. For example, given our current level of knowledge, the transformation of a "value-centered model" [6] into a "usability model" such as that of [3], can only be performed manually by designers. Semi-automation allows designers (or end-users) to adjust the models that result from transformations. For example, a designer may decide to map a subset of an AUI onto UI services developed with the latest post-WIMP toolkit. By doing so, we avoid the "low-cost, fast food" UIs as produced with automatic generation.

\footnotetext{
${ }^{2}$ As discussed in [16], comparative evaluations are more productive than absolute evaluations.
} 


\subsection{Principle \#5: Close and Open Adaptiveness Are Complementary}

Designers cannot envision all of the contexts of use in which the future interactive system will be executed. To alleviate this problem, we suggest a mix of open and close adaptiveness. A system is close-adaptive when adaptation is self-contained. It supports the "innate" adjustments planned at the design stage as well as new adjustments produced by its own internal learning mechanisms. The system is openadaptive "if new adaptation plans can be introduced during run-time" [24].

By analogy, an interactive system is close-adaptive for the contexts of use that fall within its domain of plasticity [4], that is, for the contexts of use for which this system can adapt on its own. By design, an interactive system has an innate domain of plasticity. If it is able to learn adjustments for additional contexts of use, then the domain of plasticity extends dynamically, but this extension relies only on the internal computing capabilities of the system. An external infrastructure must be provided to support open-adaptiveness [2]. Fig. 3 shows the functional decomposition of the run-time infrastructure that we propose for open model-driven adaptation.

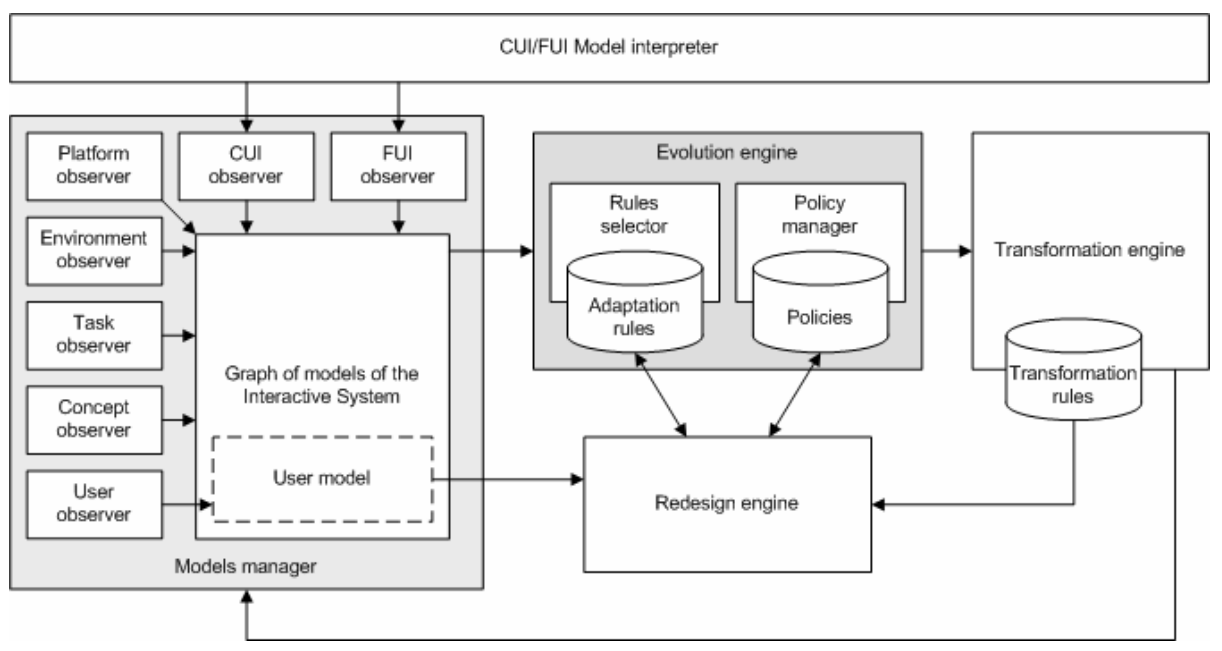

Fig. 3. Run-time infrastructure for open model-driven adaptation

Our run-time infrastructure for open model-driven adaptation includes five core functions: the Models manager, the Evolution engine, the Transformation engine, the Redesign engine and the CUI/FUI Model interpreter. For each running interactive system, the Models manager maintains the graph of models shown in Fig. 2. Observers monitor state changes in which they are interested and update the graph accordingly. Typically, a platform observer is interested in the arrival/departure of interaction resources; an FUI observer monitors the actions performed by the end-user on the FUI, etc. When some change occurs, the Models manager sends the appropriate notification to the Evolution engine or to the Redesign engine (if the change comes from the user model). The Evolution engine identifies the appropriate transformations to adapt the UI to the current change according to the current adaptation 
policy. These transformations are specified in the action part of adaptation rules. Typically, an adaptation rule obeys the Event-Condition-Action paradigm where the Event part denotes the trigger, and where the Action part makes reference to transformations or to other adaptation rules. Examples of adaptation rules will be discussed in Section 4.3. Policies serve at favoring such and such adaptation rules based on such and such (usability) criteria. The transformations selected by the evolution engine are then transmitted to the Transformation engine whose role is to apply the transformations to the graph of models. The graph of models is updated accordingly while the CUI/FUI interpreter (e.g., a Web browser) uses the new CUI to produce the new adapted final UI (FUI).

The role of the Redesign engine is to dynamically modify the adaptation rules and to set the current policy based on the information gathered from, or inferred by, the User model. Typical attributes maintained in the User model include user preferences, interests and goals, as well as interaction history (i.e. tasks in progress, completed tasks, errors, etc.) [12]. For example, the end-user using the UI shown in Fig. 1-a may keep entering wrong values when specifying a room temperature. From the information provided by the CUI/FUI observers, the User model may infer the need for error management so that the new resulting FUI be that of Fig. 1-c. The Redesign engine then searches for the transformations in the Transformations Data Base that promote the error management property. If a transformation is found, the Redesign engine may create new adaptation rules, and/or suppress adaptation rules from the current set, and/or modifies existing ones. Note that, according to Principle \#2, these modifications are performed by the way of transformations.

Having presented the principles of our approach to UI plasticity, we now show how they have been implemented and applied to HHCS.

\section{From Principles to HHCS in Action}

Our hypothesis is that designers produce all or a subset of the models mentioned in Fig. 2. For HHCS, we have adopted a top-down approach starting from the task model and the domain-dependent concepts using well-defined meta-models ${ }^{3}$. These models are represented in a simplified manner in Fig. 2. The AUI, CUI and FUI of HHCS have been produced with transformations. Examples of transformation are presented in Section 4.1. At run-time, changes in the context of use may require the user interface of HHCS to adapt. The context of use for HHCS is discussed in Section 4.2, and examples of adaptation rules are provided in Section 4.3. In Section 4.4 we show how Principle \#4 (humans are kept in the loop) is implemented with a meta-UI.

The run-time infrastructure for open model-driven adaptation is implemented as a set of OSGi services where a service encapsulates one of the core functions shown in Fig. 3. More specifically, the Models manager is implemented in Java. We draw upon the Eclipse Modeling Framework (EMF), which automatically generates the methods (the API) that make it possible for the observers and the Transformation engine to modify the graph of models. The Evolution Engine and the Redesign engine are implemented in Java as well. The Transformation engine is an ATL interpreter [1].

\footnotetext{
${ }^{3}$ Describing these meta-models would require more space than available in this paper. See [33] for more details.
} 


\subsection{Transformations Illustrated with HHCS}

Transformations, which are interpreted by the Transformation engine, are expressed in ATL [1]. QVT and XSLT are other options. The following rule (called rule TaskChoiceToGroupBox) shows how a task of type "Choose 1 item among n" (tsk.taskType.name $=$ 'Choice $1 / n$ ') is transformed into a group box using XUL as the meta-model for the expression of AUIs (XULMetaModel!GroupBox). This rule applies to a task (tsk : MMEcosystem!Task) provided that the task type is 'Choice $1 / \mathrm{n}$ ' and that there is at least one platform mapped onto the task (see the constraint tsk.taskPlatforms->select (ele.active $=$ true)->size () $>=1)$.

If the conditions are satisfied, then the transformation generates the corresponding XUL group (XULMetaModel!GroupBox) whose label is derived from the task name (capt : XULMetaModel!Caption(label <- tsk.name)) and whose content is derived from the concepts manipulated by the task. For each concept instance ( $e$ in tsk.manipulatedConcepts), the do block generates a radio button (see vbx.radiobuttons <- thisModule.radioBuild $(z)$ ) with the appropriate label (i.e., the name of the task - see XULMetaModel!RadioGroup(id <- 'radio_'+ tsk.name)). By doing so, the rule satisfies the guidance - prompting criterion defined in BastienScapin's framework.

In ATL, transformations are grouped into modules. We use the header of ATL modules to map each usability criteria with the names of the transformations that satisfy that criteria.

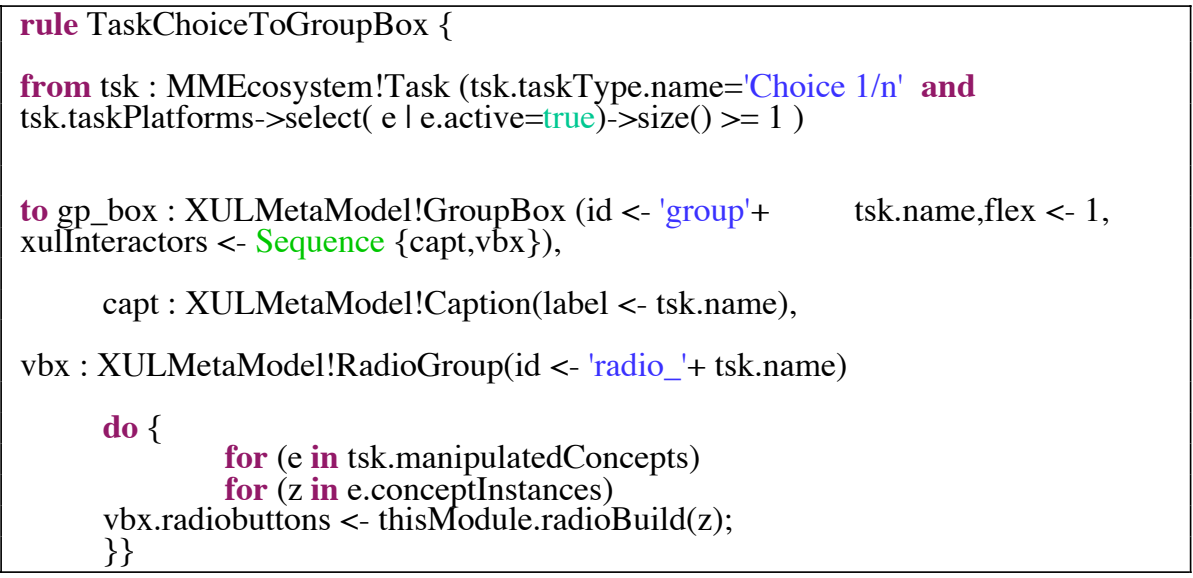

We have developed a library of transformations that can transform tasks and concept models into CUIs expressed in HTML or in XUL. As mentioned in the Principles section, transformations have also been defined to create and modify adaptation rules, which, in turn, are triggered on the occurrence of context changes.

\subsection{Context of Use in HHCS}

We model the context of use using the ontology proposed in [8]. In short, a contextual information space is modeled as a directed graph where a node denotes a context and 
an edge a condition to move between two contexts. A context is defined over a set $\mathrm{E}$ of entities, a set Ro of roles (i.e. functions) that these entities may satisfy, and a set Rel of relations between the entities. Entities, roles and relations are modeled as expressions of observables that are captured and inferred by the system. The condition to move between two contexts is one of the following: $\mathrm{E}$ is replaced with a different set, Ro has changed, or Rel has changed.

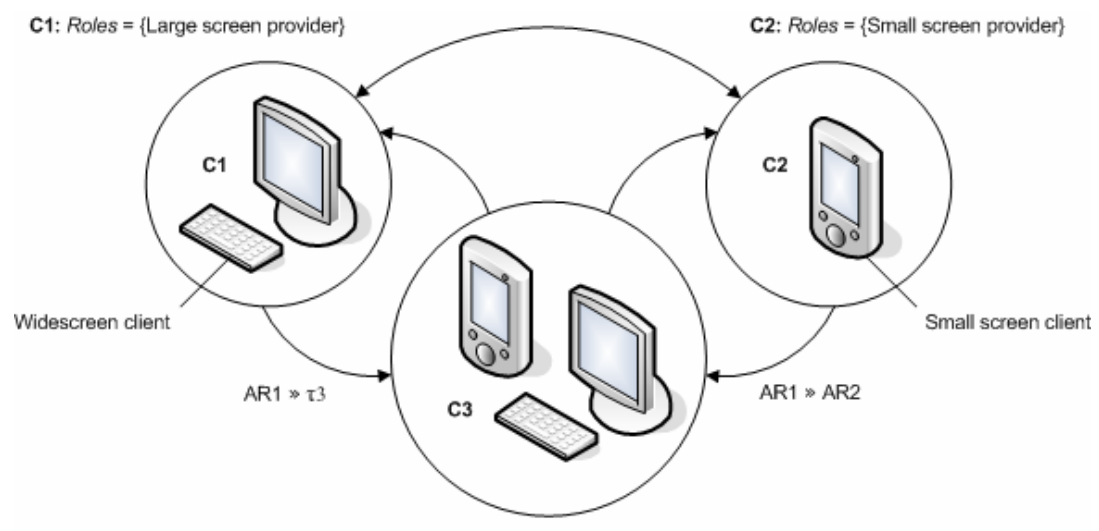

C3: Roles $=\{$ Small screen provider, Large screen provider $\}$

Fig. 4. Context switching in HHCS. Nodes denote the Roles and Entities that define a context. Arrows denote transitions between contexts decorated with the corresponding adaptation rules.

Fig. 4 shows the three contexts of use considered to be relevant for HHCS: E is the set of platforms available to the end-user, Ro the set of roles that these platforms can play (i.e. "Large screen provider" and "Small screen provider"), and where Rel is empty. $\mathrm{C} 1$ refers to the case where the user can access HHCS through a large screen whereas $\mathrm{C} 2$ enables the user to control room temperature with the small display of a PDA. In C3, a PDA and a large screen are simultaneously available. As discussed above, the arrival/departure of a platform is detected by the platform observer: the platform model is modified accordingly in the Models manager, and a "platform modification" event is sent to the Evolution engine. Events serve as triggers for the adaptation rules interpreted by the Evolution Engine.

\subsection{Adaptation Rules}

Adaptation Rules (AR) comply with the meta-model shown in Fig. 5. This metamodel re-uses the classical Event-Condition-Action structure, with additional Pre- and Post-conditions:

- The Event and conditions (Condition, Pre- and Post-conditions) make reference to the models maintained in the Models manager; they are possibly empty.

- The Action references either the model transformation or the adaptation rule (AR) to be applied. The action may satisfy (or may not satisfy) a set of properties (for instance, the Bastien-Scapin's criteria). 


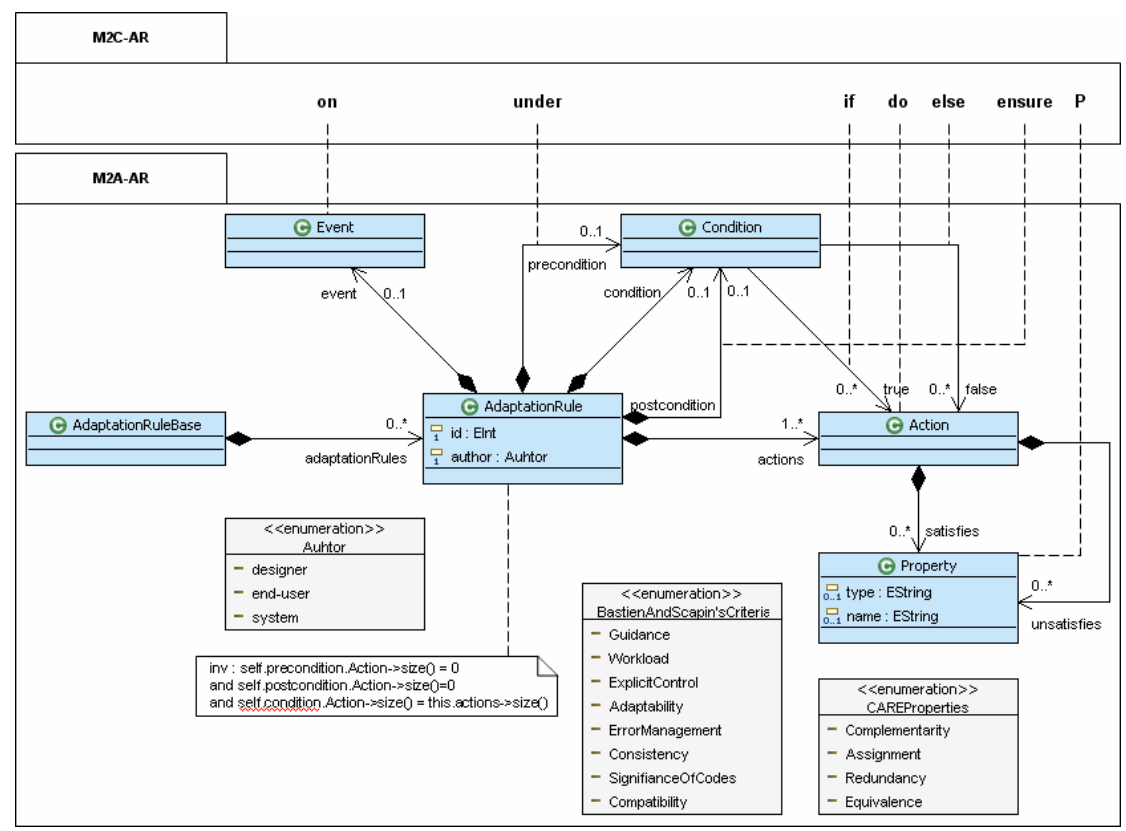

Fig. 5. Our meta-model for adaptation rules

The following adaptation rules have been defined to support the changes in the context of use depicted in Fig. 4:

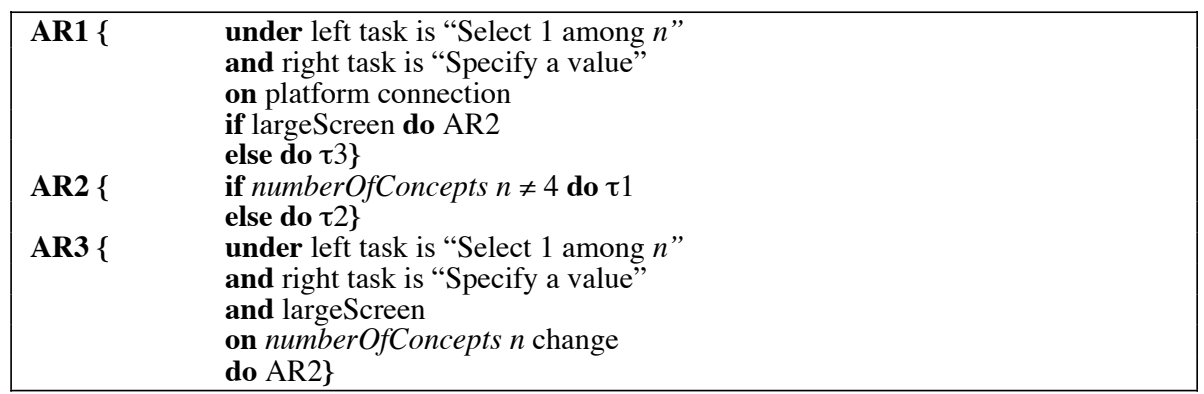

AR1 is triggered on the connection of a new platform and applies to tasks, like task "Set home temperature" of Fig. 1, whose first sibling consists in choosing 1 item among $\mathrm{n}$, and the second sibling consists in specifying a value. AR1 invokes Transformation $\tau 3$ when the role "Large screen provider" is not satisfied. It invokes AR2 otherwise. AR2 calls for Transformation $\tau 2$ when the number of concepts referenced in the task is four, leading to the generation of the final UI shown in Fig. 1-b. This transformation, which avoids articulatory tasks, supports the "minimal action" criteria.

AR3 is triggered by the event "numberOfConcepts n change". In HHCS, this corresponds to the case where a new thermostat is installed/removed from the home. In 

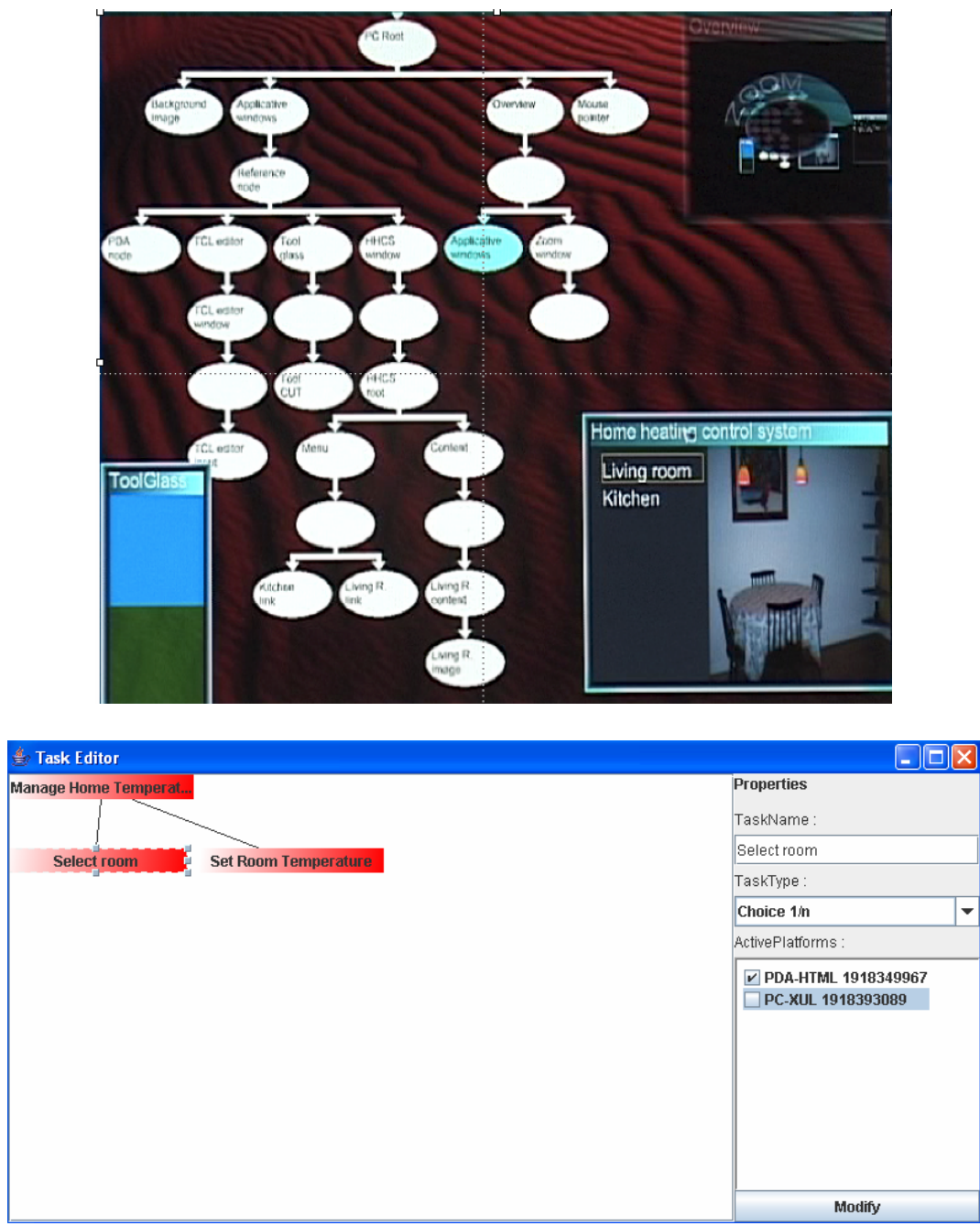

Fig. 6. Examples of meta-UIs when controlling the FUI of HHCS

HHCS, this phenomenon is detected by the concept observer. The concept model is modified accordingly, and the corresponding event is sent to the Evolution engine. AR3 is applied when the role "Large screen provider" is filled.

When several adaptation rules apply, the Policy Manager makes the final decision based on the current active policy and provides the Transformation Engine with the appropriate transformations. Interoperability between the Evolution Engine and the ATL-based Transformation Engine is ensured in the following way: Adaptation rules are produced using an EMF (Eclipse Modeling Framework) basic editor. The output of this editor is an XMI file (.ecore) that can be manipulated by EMF-based tools such as our ATL Transformation Engine. 
So far, we have shown how the run-time infrastructure supports run-time adaptation based on the specifications provided by the designers. In the next section, we show how the end-user (and the designer) can be kept in the loop at run-time (Principle \#4) when served by a meta-UI.

\subsection{Meta-UIs to Keep Humans in the Loop at Run-Time}

A meta-UI is an interactive system whose set of functions is necessary and sufficient to control and evaluate the state of an interactive ambient space [9]. This set is metabecause it serves as an umbrella beyond the domain-dependent services that support human activities in this space. It is $U I$-oriented because its role is to allow users to control and evaluate the state of the ambient interactive space. It is to ambient computing what desktops and shells are to conventional workstations.

Fig. 6 shows early versions of meta-UIs illustrated on HHCS. In the top example, the CUI model of HHCS as well as that of the meta-UI is a scene graph rendered as the display background. A subtree of the CUI is suppressed by bringing the tool-glass on the root of the subtree followed by a click through. The FUI is updated accordingly. Conversely, the user modifies the FUI with the tool-glass and the CUI is updated accordingly. In the bottom example, end-users can map the tasks "Select room" and "Set room temperature" respectively, to the PDA-HTML platform and to the PCXUL platform, resulting in the FUI shown in Fig. 1-e. These toy examples are being redesigned to fully exploit the flexibility provided by our approach (as well as to improve their usability!).

\section{Conclusion}

Our approach addresses the complexity of UI plasticity with a combination of MDE and SOA. In this paper, the focus is set on MDE, but the running example combines both of them. Model-based generation has received little acceptance due to the high threshold of learning new languages for a low pay-off (obtaining simplistic UIs). In response to this limitation, we propose the use of transformations as models that can be capitalized in re-usable libraries. Furthermore, we allow hand-coded fine-tuned portions of a UI (such as the toolglass of our meta-UI) to be dynamically mapped onto high-level models provided that they comply with a component-oriented service protocol (this aspect has not been discussed in the paper). Transformations are key because they can be dynamically transformed, either automatically by a run-time infrastructure and/or by the end-users and designers through a meta-UI. Transformations can also be used to improve interoperability. In particular, we have defined transformations that translate our own meta-models into UsiXML [14] meta-models, so as to take benefit from the UsiXML arsenal of tools.

A subset of our principles has been applied to a simplified version of a Home Heating Control System. Other applications are under way for SMS and ambient computing to support conference participants in large conferences. Although this serves as our validation for this work, it also leaves several opportunities for improvements including the study of end-user development environments such as the concept of meta-UI. 
Acknowledgments. This work has been partly supported by Project EMODE (ITEAif4046), the NoE SIMILAR- FP6-507609, and France-Télécom R\&D.

\section{References}

1. ATL : Atlas Transformation Language, http://www.eclipse.org/m2m/atl/

2. Balme, L., Demeure, A., Barralon, N., Coutaz, J., Calvary, G.: CAMELEON-RT: a Software Architecture Reference Model for Distributed, Migratable, and Plastic User Interfaces. In: Markopoulos, P., Eggen, B., Aarts, E., Crowley, J.L. (eds.) EUSAI 2004. LNCS, vol. 3295, pp. 291-302. Springer, Heidelberg (2004)

3. Bastien, J.M.C., Scapin, D.: Ergonomic Criteria for the Evaluation of Human-Computer. Technical report INRIA, No 156 (June 1993)

4. Calvary, G., Coutaz, J., Thevenin, D.: A Unifying Reference Framework for the Development of Plastic User Interfaces. In: Nigay, L., Little, M.R. (eds.) EHCI 2001. LNCS, vol. 2254, pp. 173-192. Springer, Heidelberg (2001)

5. Clerckx, T., Luyten, K., Coninx, K.: Generating Context-Sensitive Multiple Device Interfaces from Design. In: Proceedings of CADUI'2004, Isle of Madeira, pp. 288-301 (2004)

6. Cockton, G.: A Development Framework for Value-Centred Design. In: Extended Abstracts Proc. of CHI 2005, Portland, Oregon, USA, April 2-7, 2005, pp. 1292-1295 (2005)

7. Constantine, L.L., Lockwood, L.A.D.: Software for Use: A Practical Guide to the Models and Methods of Usage-Centred Design. Addison-Wesley, New-York (1999)

8. Coutaz, J., Crowley, J.L., Dobson, S., Garlan, D.: Context is Key. Communication of the ACM (CACM) 48(3), 49-53 (2005)

9. Coutaz, J.: Meta-User Interfaces for Ambient Spaces. In: Coninx, K., Luyten, K., Schneider, K.A. (eds.) TAMODIA 2006. LNCS, vol. 4385, pp. 1-15. Springer, Heidelberg (2007)

10. Dix, A., Finlay, J., Abowd, G., Beale, R.: Human-Computer Interaction. Prentice-Hall, New-Jersey (1993)

11. Gajos, K., Weld, D.: SUPPLE: Automatically Generating User Interfaces. In: Proceedings of the 9th international conference on Intelligent User Interfaces, pp. 93-100. ACM Press, New York (2004)

12. Johansson, P.: User Modeling in Dialog Systems. Santa Anna IT Research Institute Report SAR (2002)

13. Kurtev, I., Bézivin, J., Aksit, M.: Technological Spaces: An Initial Appraisal. In: Meersman, R., Tari, Z., et al. (eds.) CoopIS 2002, DOA 2002, and ODBASE 2002. LNCS, vol. 2519, Springer, Heidelberg (2002)

14. Limbourg, Q., Vanderdonckt, J., Michotte, B., Bouillon, L., Lopez, V.: USIXML: a Language Supporting Multi-Path Development of User Interfaces. In: Bastide, R., Palanque, P., Roth, J. (eds.) Engineering Human Computer Interaction and Interactive Systems. LNCS, vol. 3425, pp. 200-220. Springer, Heidelberg (2005)

15. Lopez-Jaquero, V., Montero, F., Molina, J.P., Gonzalez, P.: A Seamless Development Process of Adaptive User Interfaces Explicitly Based on Usability Properties. In: Bastide, R., Palanque, P., Roth, J. (eds.) Engineering Human Computer Interaction and Interactive Systems. LNCS, vol. 3425, pp. 289-291. Springer, Heidelberg (2005)

16. Molich, R., Ede, M., Kaasgaard, K., Karyukin, B.: Comparative usability evaluation. Behaviour \& Information Technology 23(1), 65-74 (2004)

17. Montero, F., Vanderdonckt, J., Lozano, M.: Quality Models for Automated Evaluation of Web Sites Usability and Accessibility. In: Koch, N., Fraternali, P., Wirsing, M. (eds.) ICWE 2004. LNCS, vol. 3140, pp. 28-30. Springer, Heidelberg (2004) 
18. Mori, G., Paternò, F., Santoro, C.: Design and Development of Multidevice User Interfaces through Multiple Logical Descriptions. IEEE Transactions on Software Engineering 30(8), 507-520 (2004)

19. Myers, B., Hudson, S.E., Pausch, R.: Past, Present and Future of User Interface Software Tools. Transactions on Computer-Human Interaction (TOCHI) 7(1), 3-28 (2000)

20. Nichols, J., Rothrock, B., Chau, D., Myers, B.: Huddle: Automatically Generating Interfaces for Systems of Multiple Connected Appliances. In: Proc. of UIST'2006, pp. 279-288 (2006)

21. Nielsen, J.: Heuristic evaluation. In: Nielsen, J., Mack, R.L. (eds.) Usability Inspection Methods, John Wiley \& Sons, New York (1994)

22. Njike, H., Artières, T., Gallinari, P., Blanchard, J., Letellier, G.: Automatic learning of domain model for personalized hypermedia applications. In: IJCAI. International Joint Conference on Artificial Intelligence, Edinburg, Scotland, pp. 1624-1625 (2005)

23. Nóbrega, L., Nunes, J.N., Coelho, H.: Mapping ConcurTaskTrees into UML 2.0. In: Gilroy, S.W., Harrison, M.D. (eds.) Interactive Systems. LNCS, vol. 3941, pp. 237-248. Springer, Heidelberg (2006)

24. Oreizy, P., Gorlick, M., Taylor, R., Heimbigner, D., Johnson, G., Medvidovic, N., Quilici, A., Rosenblum, D., Wolf, A.: An Architecture-Based Approach to Self-Adaptive Software. IEEE Intelligent Systems 14(3), 54-62 (1999)

25. Paternò, F.: Model-Based Design and Evaluation of Interactive Applications. Springer, Heidelberg (2000)

26. Preece, J., Rogers, Y., Sharp, H., Benyon, D., Holland, S., Carey, T.: Human-Computer Interaction. Addison Wesley Publ., Wokingham, UK (1994)

27. Seffah, A., Donyaee, M., Kline, R.B.: Usability and quality in use measurement and metrics: An integrative model. Software Quality Journal (2004)

28. Shackel, B.: Usability - Context, Framework, Definition, Design and Evaluation. In: Human Factors for Informatics Usability, pp. 21-38. Cambridge University Press, Cambridge (1991)

29. Sheshagiri, M., Sadeh, N., Gandon, F.: Using Semantic Web Services for Context-Aware Mobile Applications. In: Proc. of ACM MobiSys2004 Workshop on Context Awareness, Boston, Massachusetts, USA, ACM Press, New York (2004)

30. Shneiderman, B.: Designing User Interface Strategies for effective Human-Computer Interaction, 3rd edn., p. 600. Addison-Wesley Publ, Reading (1997)

31. da Silva, P.: User Interface Declarative Models and Development Environments: A Survey. In: Proc. of DSV-IS2000, June 5-6, 2000, pp. 207-226. Springer, Limerick, Ireland (2000)

32. Schmidt, D.C.: Guest editor's introduction: Model-Driven Engineering. IEEE Computer 39(2), 25-31 (2006)

33. Sottet, J.S., Calvary, G., Coutaz, J., Favre, J.M.: A Model-Driven Engineering Approach for the Usability of Plastic User Interfaces. In: EIS'07. the proceedings of Engineering Interactive Systems 2007, March 22-24, 2007, pp. 22-24. University of Salamanca, Spain (2007)

34. Thevenin, D., Coutaz, J.: Plasticity of User Interfaces: Framework and Research Agenda. In: Proc. Interact99, Edinburgh, pp. 110-117. IFIP IOS Press Publ. (1999)

35. Van Welie, M., van der Veer, G.C., Eliëns, A.: Usability Properties in Dialog Models. In: DSV-IS'99. 6th International Eurographics Workshop on Design Specification and Verification of Interactive Systems, Braga, Portugal, 2-4 June 1999, pp. 238-253 (1999) 